\title{
Longitudinal Inter-vehicle Distance Control of Autonomous Vehicle Platoons Subjected to Internal and External Disturbances
}

\author{
Alex Gunagwera and Aydin Tarik Zengin*
}

\begin{abstract}
The idea of autonomous vehicle platoons presents a variety of social, economic, and safety benefits to the transportation industry. However, implementing and deploying autonomous vehicle platoons is still a challenge. In this paper, we present a PID-based computationally cost-efficient controller to aid in the longitudinal control of the inter-vehicle distance between successive platoon members. The proposed approach is facilitated by inter-vehicle communication. The algorithm was implemented using the Robotics Operating System and Gazebo simulation environment. In order to evaluate the performance and applicability of the proposed approach, meticulous simulations under numerous scenarios were performed using 3D vehicle models so as to mimic the real-world. The algorithm successfully maintains the longitudinal inter-vehicle distance within the desired range, ensures that no collisions occur among platoon members, and preserves the platoon formation.
\end{abstract}

Index Terms-Autonomous vehicles, autonomous vehicle platoons, intelligent transport systems, longitudinal platoon control, automated highway systems, PID.

\section{INTRODUCTION}

A UTONOMOUS VEHICLE PLATOONS (AVPs) is a trending multidisciplinary topic nowadays attracting attention from researchers, practitioners, and governmental bodies all over the world. AVPs are comprised of two or more vehicles mechanically or electronically connected and travelling closely together as a single unit with the same lateral and, or longitudinal motion control. Autonomous vehicle platooning promises a variety of social, economic and safety benefits ranging from saving employee time [1], optimal energy consumption [2], efficient road utilization [3]

ALEX GUNAGWERA, is with Department of Computer Engineering Istanbul Sabahattin Zaim University, Istanbul, Turkey, (e-mail: alex.gunagwera@izu.edu.tr).

iD https://orcid.org/0000-0002-0143-3743

AYDIN TARIK ZENGIN, is with Department of Computer Engineering Istanbul Sabahattin Zaim University, Istanbul, Turkey, (e-mail: tarik.zengin@izu.edu.tr).

iD https://orcid.org/0000-0002-0860-4509

Manuscript received Nov 1, 2021; accepted Jan 28, 2022.

DOI: $10.17694 /$ bajece.1017623 to minimizing traffic accidents culminating from human error [4].

Owing to the multidisciplinary nature of AVPs, a multitude of work has been done pertaining to AVPs; their control, analysis, deployment to mention but a few. Work from areas such as control and analysis, the communication industry, and energy department have contributed significant work to the enhancement of AVP applications. For instance, studies on Adaptive Cruise Control (ACC) [5], Cooperative Adaptive Cruise Control (CACC) [6], [7], String Stability [8], [9]. The cruise control model proposed by [5] was implemented in MATLAB SIMULINK. They used the velocity and intervehicle distance deviation as inputs to their controller.

Communication plays a vital role in the overall success of autonomous vehicle platoons. In fact, AVPs incorporating communication in their architecture register better results in comparison to those that operate without communication among platoon members [10]. The quality, ease of communication and type of information shared significantly affects the performance of an AVP. Furthermore, the efficiency of the communication methodology employed, and the amount of information communicated in the platoon also have the ability to enhance the success of the entire platoon. Steven E. Shladover et al. [6] provided the essential definitions and distinctions among the different types of CACC and the various communication types employed by platoons. Robust Vehicle to Vehicle (V2V) communication, such as VANET, DSRC [11], [12], Vehicle to Infrastructure (V2I) or even both (V2X) communications facilitate the functioning of AV platooning. Shen, Z. et al. [13] discussed the effects of communication reliability and latency on the performance of vehicle systems using 5G V2X hardware prototypes and the 802.11 communication protocol. In our study, we utilize the Robot Operating System (ROS) [14] messages and topics for communication within the platoon. Inter vehicle communication is wireless and is based on WiFi(IEEE 802.11) network.

String stability is another important feature of an autonomous vehicle platoon. Cremer, D. [9] provided a string stability criterion which only depends on the error in velocity of each vehicle in comparison to the velocity of the Leading Vehicle $(L V)$. Cremer's standards did not rely on the intervehicle distance. Seiler et al. defined platoon stability as the error between the desired and the actual inter-vehicle spacing [15]. Swaroop et al. [16] presented string stability requirements that depend on the inter vehicle distance in two 
categories: the strong sense and the weak sense. In the strong sense, the presented string stability conditions requires that the maximum inter vehicle distance error of the $i^{\text {th }}$ vehicle should either be equal or less to that of the $i-1^{\text {th }}$ vehicle. String stability in a weak sense has a requirement that just the maximum inter-vehicle distance errors should be less than or equal to those of the first follower $(F)$ vehicle.

The Global Positioning System (GPS) [17], is one of the most valuable sensors in look-ahead systems. For example, [18] illustrated a high integrity navigation system's development and implementation for usage in autonomous land vehicle applications. They mainly used GPS and the Inertial Measurement Unit (IMU) in their work. GPS is the backbone of the approach we present in this study as well.

Inter-vehicle distance (IVD) is an essential metric in autonomous vehicle platoons. How well it is kept and maintained ensures safe, comfortable, and more efficient road usage, among others. It is with this background that we propose this study.

In this paper, we propose a computationally cost efficient, PID-based algorithm for controlling the inter vehicle distance between successive members of an autonomous vehicle platoon. Our approach differs from other numerous studies mainly by using only the onboard GPS sensors of the vehicles, gazebo robot simulator and the Robot operating system (ROS). Furthermore, our PID controller requires just the current longitudinal inter vehicle distance to the preceding vehicle, unlike most PID approaches that require the velocity and acceleration information as well. The algorithm observes maintenance of platoon formation and makes sure no collisions occur amongst platoon members. Obtained results are presented and 3D simulations of the system further carried out using ROS and Gazebo platforms to demonstrate the performance of the proposed approach.

This paper is organized as follows. Section II presents the problem statement and followed platoon model. Section III explains the scenarios considered during simulations. Section IV presents the environment and conditions under which the simulations were performed. Section V illustrates the results obtained from the simulations, whereas Section VI discusses the obtained results, the limitations of the proposed approach, overall practicality, and applicability of the proposed algorithm. Furthermore, how the proposed algorithm differs from the current related works. Finally, in Section VII, we conclude this work and present directions for the future work.

\section{PRoblem Statement And Platoon Model}

The controlled platoon comprises of four vehicles in total. The platoon Leader Vehicle $(L V)$ and three Follower $(F)$ vehicles. We design a PID controller to aid the control of the distance between vehicles. It takes as input the current inter vehicle distance between vehicles and returns as output a velocity reference for the corresponding $F_{i}$ vehicle in order to achieve the desired inter-vehicle distance, (D) to the preceding vehicle. We thus state the problem as:

$$
\begin{gathered}
\operatorname{Set}\left(d_{i}\right)=D \pm\left|E_{i}\right| \\
E_{i}=D-d_{i}
\end{gathered}
$$

and $\left|E_{i}\right| \leq E_{\text {thresh }}$.

Ultimately, the major purpose of our PID controller is to reduce the error, $E_{i}$, and drive it as close to $0 m$ as possible. So, the best-case scenario at any point in the simulation is to have $E_{i}=0 m$, especially during the steady state. $\forall F_{i}, i \in\{1,2,3\}$ where $d_{i}$ is the $i^{\text {th }}$ inter-vehicle distance, $D$ is the desired intervehicle distance, and $E_{i}$ is the error between the $i^{\text {th }}$ intervehicle distance and the desired distance, $D$. $E_{\text {thresh }}$ is the maximum and minimum threshold value beyond which the error should not exceed in order to guarantee safety. This constraint ensures that $F$ vehicles are not allowed to fall more than $E_{\text {thresh }}$ behind the preceding vehicle, i.e., $E_{i} \leq E_{\text {thresh }}$. It also ensures that $F$ vehicles do not get more than $E_{\text {thresh }}$ closer to the preceding vehicle, i.e., $E_{i} \geq-E_{\text {thresh }}$. Every $F$ vehicle runs its own instance of the PID control algorithm. The platoon model presented in this work is based on the PredecessorFollower communication model [19]. In the simulations, the desired inter-vehicle distance, $D$, is set to $12 \mathrm{~m}$ and $E_{\text {thresh }}$ is considered as $5 m$.

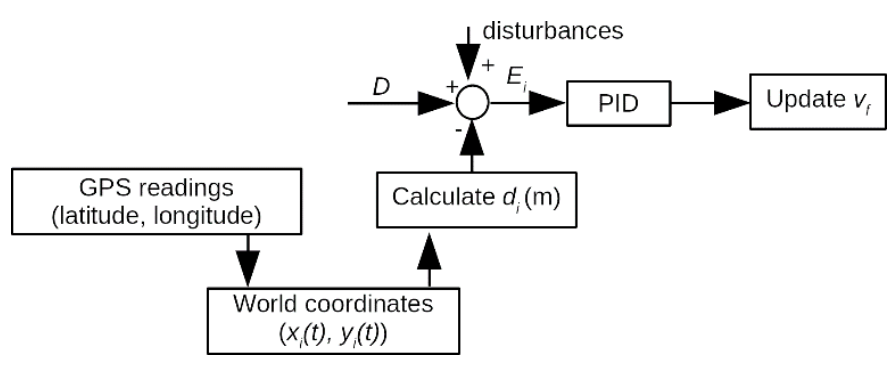

Fig.1. Controller Model

Fig.2. demonstrates the platoon setup. $L V$, is the Leading vehicle, also referred to as the root node of the platoon and is generally indexed as the first member of the platoon. The $F$ labels depict the follower vehicles. The distance from one vehicle's center of mass to the preceding vehicle's center of mass is referred to as the inter-vehicle gap/distance in this study.

In this study, the control algorithm aims at ascertaining a constant inter-vehicle gap with all vehicles' velocity sufficiently approximately equal to that of the LV in the platoon using only the distance measure from the data provided by the onboard GPS sensors of the vehicles. Errors in the inter-vehicle gap should be bounded, and there should be always no collisions among platoon members in the worstcase scenario during the simulation. Following the definition of the platoon stability provided by [15], we can formulate the steady-state error transfer function as

$$
H(s)=\frac{E_{i}}{E_{i-1}}
$$

Followingly, platoon stability is guaranteed, locally, if $\|H(s)\|_{\infty} \leq 1$, and $h(t)>0$ where $h(t)$ gives the impulse response corresponding to $H(s)$ as per the $\zeta_{2}$ norm [10]. $\zeta_{\infty}$ extends this notion throughout the whole platoon to ensure that overshoots do not occur as the signals propagate up the string, hence global stability. 
C. 0 0

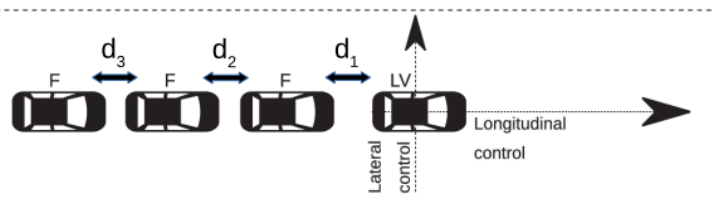

Fig.2. Illustration of inter-vehicle distance, di, LV, and $\mathrm{F}$

At the start of the simulation, GPS data measurements are retrieved asynchronously if available from every vehicle's onboard GPS sensor. From this data, the relative inter-vehicle gap is calculated and forwarded to the PID algorithm which in turn returns the reference velocity with which the corresponding $F$ vehicle's velocity is updated to achieve the desired inter-vehicle gap between $F$ and the preceding vehicle. The desired inter-vehicle distance, $D$ is set as the PID algorithm's setpoint whereas the inter-vehicle gap estimates, calculated from the data measurements provided by the GPS sensor, is provided as the feedback for the PID control algorithm. We repeat these steps throughout the simulation lifetime.

Fig.1. shows the general algorithm flow. Each $F_{i}$ vehicle runs this algorithm with $v_{f}$ being its effective reference velocity.

\section{CONSIDERED Scenarios DuRING Simulations}

In reality, systems are exposed to various conditions and sensors do not perform as smoothly as intended. Due to natural and mechanical phenomena, sensor readings are generally, corrupted and affected by external noise. Furthermore, there is exhibited by the vehicle onboard sensors (sensor lag), lag within the vehicle control system and the delay in $\mathrm{V} 2 \mathrm{~V}$ communication. These are all inevitabilities that should be put into consideration if we wish to make the simulations as realistic, and accurate as possible. In case, the LV is either being manned by a human or is following a human-manned vehicle in traffic, the human-factor may, to some degree, not be negligible, i.e., the driver may suddenly brake or accelerate for any uncertain period of time. We shall, hereafter, refer to this phenomenon as the human factor $(H F)$ which may or may not be present in a particular scenario.

In this section we present the performance of the proposed controller with the above-mentioned criteria under consideration. The simulated scenarios are divided into four categories: In the first scenario, the platoon is only affected by sensor lag. $H F$ and other forms of delays discussed are not present. In the second scenario, the platoon is subjected to both the sensor lag and random occurrences of the $H F$, only. In the third scenario, the platoon is subjected to the sensor lag, random V2V communication, and vehicle control delays with no $H F$ occurrences. In the final scenario, the platoon is subjected to the random $H F$, sensor lag, and random V2V communication, and control delays. For all simulation scenarios presented in this section, the $L V$ action at any given moment can be one of acceleration, deceleration or moving at constant speed. The action is randomly generated with the following limitations and constraints:

1) Reverse vehicle motion is not permitted within the platoon. In case the LV velocity, during the deceleration phase period, were to drop below $0 \mathrm{~m} / \mathrm{s}$, the $\mathrm{LV}$ is programmed to stop, i.e., $\mathrm{LV}$ velocity is set to $0 \mathrm{~m} / \mathrm{s}$.

2) No action; acceleration, deceleration or constant speed should be executed more than once consecutively. This way, we ensure that the platoon performance under various uncertain scenarios is observed.

3) The duration of each phase/action is randomly generated and may last between 20 - 50 seconds with the exception of the final phase - the phase during which the LV decelerates to rest.

The first and final phases are a bit different. Since the platoon starts motion from rest, the first phase/action has to always be acceleration; the $L V$ accelerates for a random period of time. The final phase is always the deceleration of the $L V$ to rest, therefore its duration is not determined randomly. The vehicle simply uniformly decelerates to $0 \mathrm{~m} / \mathrm{s}$.

Table 1 provides a categorical summary of the scenarios considered in this simulation. Follower vehicle controllers are data from sensors. The sensors are subjected to reductions in update frequency which leads to delays in the processed data. This helps to portray unfavorable real-world conditions [21].

$\mathrm{V} 2 \mathrm{~V}$ communication frequency was set in such a way that vehicles publish their information at an average of $33 \mathrm{~Hz}$, which is the proposed maximum frequency by [20]. We vary this frequency with a minimum being $20 \mathrm{~Hz}$ and the maximum being $50 \mathrm{~Hz}$ to account for variations in platoon member mechanical differences as well as miscellaneous occurrences such as natural phenomena such as variations in wind speeds. The time between messages varies with an average of $0.03 \mathrm{~s}$ with a minimum of $0.02 \mathrm{~s}$ and a maximum of $0.05 \mathrm{~s}$.

TABLE I

SUMMARY OF THE SCENARIOS OF THE SCENARIOS CONSIDERED DURING SIMULATIONS

\begin{tabular}{|l|l|}
\hline Scenarios 1 and 2 & Scenarios 3 and 4 \\
\hline sensor lag w/o $H F$ & sensor lag, V2V communication delays, \\
w/o $H F$ \\
sensor lag w/HF & $\begin{array}{l}\text { sensor lag, V2V communication delays, } \\
\text { w/HF }\end{array}$ \\
\hline
\end{tabular}

\section{Simulation EnVironment}

3D vehicles were designed and modeled using Gazebo robotics simulator version 9 integrated with ROS 1 (Melodic). Visuals of the 3D vehicle objects were designed using the gazebo platform, whereas vehicle motion was handled and controlled via topics and messages by the nodes implemented using the ROS framework [14]. The vehicle model is based on the Hyundai Genesis-2014 [20] which reproduces the major dynamic characteristics such as friction, acceleration, deceleration/braking, wheel radius, and weight making it possible to critically analyze the movement of vehicles in the platoon. 


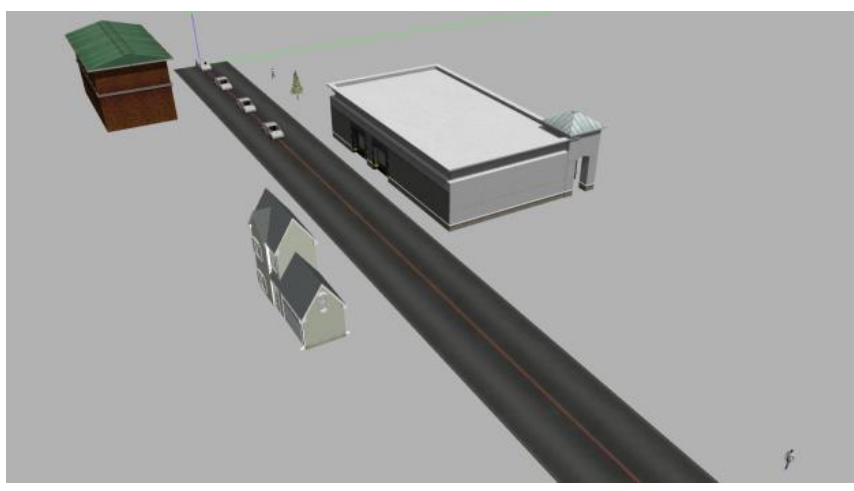

Fig.3. Gazebo simulation environment

Table 2 presents some of the major vehicle model parameters and their values.

TABLE II
\begin{tabular}{|l|l|} 
VEHICLE INFORMATION \\
\hline Vehicle Attribute & Value \\
\hline Vehicle Mass & $1823.0 \mathrm{~kg}$ \\
Vehicle Length & $5 \mathrm{~m}$ \\
Vehicle Width & $1.89 \mathrm{~m}$ \\
Vehicle Height & $1.480 \mathrm{~m}$ \\
Wheel Radius & $0.34 \mathrm{~m}$ \\
Wheelbase & $2.95 \mathrm{~m}$ \\
Wheel Width & $0.225 \mathrm{~m}$ \\
Drag coefficient & $0.27 \mathrm{~cd}$ \\
\hline
\end{tabular}

Fig.3. shows the environment in which the simulations were performed and monitored. The vehicles move forward along the road during simulation to preserve the presented platoon formation and the desired inter-vehicle distance. We performed simulations under the assumptions and constraints that:

1) The roads are straight and have got no slope so that longitudinal control of the platoon remains the focus of the platoon

2) Communication is wireless, that is, over a Wi-Fi (IEEE 802.11) and each vehicle is only allowed to communicate to the preceding vehicle.

3) Overtake and reversing manoeuvres are not allowed in the platoon.

\section{RESULTS}

In this section we present the results obtained from the performed simulations. For each simulation scenario, two study cases are presented. We manually tuned the PID controller. The parameter gains of the controller that yielded the presented results are listed in Table 3. We conclude from the observed results that the proposed approach indeed does guarantee the following effect while keeping the inter-vehicle distance within the desired range. The algorithm also ensures platoon formation preservation and no collisions amongst platoon members since the error, $E_{i} \geq-E_{\text {thresh }}$ at all times throughout the simulation. However, much as the vehicles in the platoon obtain vehicle stability [23], overall platoon stability is not guaranteed. The proposed controller also shows best performance at steady state when the $L V$ travels with uniform velocity - as can be most explicitly observed in the first scenario of the simulations. All error values presented include the transient errors.

TABLE III
PID CONTROLLER PARAMETERS
\begin{tabular}{|l|l|}
\hline Parameter & Value \\
\hline$P$ & 0.05 \\
$I$ & 0.00001 \\
$D$ & 0.03 \\
\hline
\end{tabular}

Long et al. [24] proposed a distributed model predictive model for the longitudinal control of truck platoons. Their model considers the state of the $L V$. In their study, platoon members transition from cruise control (CC), adaptive cruise control (ACC), and cooperative cruise control(CACC). ACC and $\mathrm{CACC}$ constitute the most advanced platoon controllers that exist today. They use MATLAB to evaluate the performance of their model. We present a summarized comparison of the performance of our approach and the ACC phase of their study. Throughout the simulations, Long et al. had the LV accelerate to $\approx 16 \mathrm{~m} / \mathrm{s}$, then move with constant velocity after that. They experienced a maximum transient response error of $\approx 37 \mathrm{~m}$ before the system finally settled and had the range error converge to $0 \mathrm{~m}$. Our proposed controller had a maximum transient error of $4.9 \mathrm{~m}$, after which it converged to or close to $\mathrm{Om}$ at steady state especially whenever the $L V$ was moving with constant speed.

\section{A. Scenario 1}

In this scenario, platoon members were only subjected to sensor lag. This lag is assumed to occur in the GPS sensors in this study. This scenario registered the best performance. In this scenario, global platoon stability can seldomly be observed at steady state especially when the $L V$ travels with constant speed.

In the first case study of scenario 1 (Fig.4.), there is the $L V$ velocity throughout the simulation illustrated in the first subfigure. Platoon real-time IVD $\left(d_{i}\right)$ is presented in the second subfigure. The third subfigure presents the error $\left(E_{i}\right)$ in the IVD in comparison to the desired IVD $(D)$. The fourth subfigure presents platoon members' real-time velocity profiles during the simulation. In the first and second subfigures, blue denotes the IVD $\left(d_{l}\right)$ and error $\left(E_{l}\right)$ in IVD between the $L V$ and $F_{1}$, respectively. Similarly, red and green, denote the IVD $\left(d_{2}, d_{3}\right)$ and their corresponding errors $\left(E_{2}, E_{3}\right)$, respectively. In the fourth subfigure, $v 1$, depicted by blue, shows the LV velocity while v2(red), v3(green) and v4(cyan) represent the velocities of the $F$ vehicles (1, 2, and 3), respectively.

During this case study, a minimum error of $-2.8 m$ and a maximum error of $3.0 \mathrm{~m}$ in IVD were obtained with maximum standard deviation and variance of $1.94 \mathrm{~m}$ and $3.7 \mathrm{~m}^{2}$, respectively. During this scenario, local platoon stability is not guaranteed since, for instance, $E_{3} / E_{2}>1$.

Similarly, in the second case study of the first scenario (Fig. 5.), there is the $L V$ velocity throughout the simulation. Platoon real-time IVD $\left(d_{i}\right)$ is presented in the second subfigure. The 

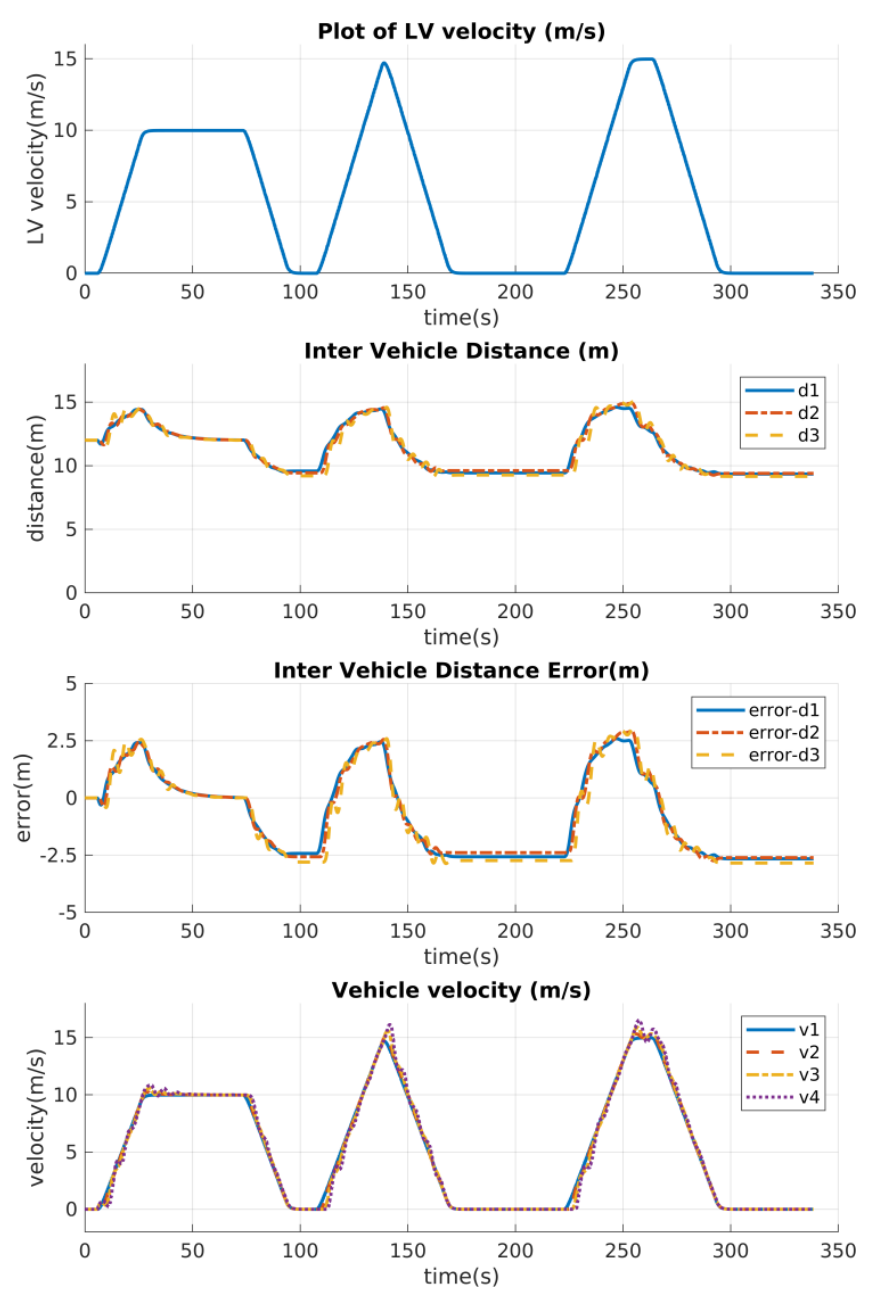

Fig.4. Scenario 1, first case study

third subfigure presents the error $\left(E_{i}\right)$ in the IVD in comparison to the desired IVD $(D)$. The fourth subfigure presents platoon members' real-time velocity profiles during the simulation. In the first and second subfigures, blue denotes the IVD $\left(d_{l}\right)$ and error $\left(E_{l}\right)$ in IVD between the $L V$ and $F_{l}$, respectively. Similarly, red and green, denote the $\operatorname{IVD}\left(d_{2}, d_{3}\right)$ and their corresponding errors $\left(E_{2}, E_{3}\right)$, respectively. In the fourth subfigure, $v 1$, depicted by blue, shows the LV velocity while v2 (red), v3 (green) and v4 (cyan) represent the velocities of the $F$ vehicles (1-3), respectively. In this particular case study, a minimum error of $-3.5 \mathrm{~m}$ and a maximum error of $4.2 \mathrm{~m}$ in IVD were obtained with maximum standard deviation and variance of $1.9 \mathrm{~m}$ and $4.9 \mathrm{~m}^{2}$, respectively. During this scenario, local platoon stability is not guaranteed since, for instance, $E_{2} / E_{1}>1$.

\section{B. Scenario 2}

In this scenario, the platoon members were subjected to sensor lag and the $L V$ was subjected to random $H F$.

In the first subfigure of the Scenario 2's first case study(Fig. 6.), we have the $L V$ velocity profile during the simulation. Platoon real-time $\operatorname{IVD}\left(d_{i}\right)$ in the second subfigure followed by the error $\left(E_{i}\right)$ in the IVD in comparison to the desired IVD $(D)$,
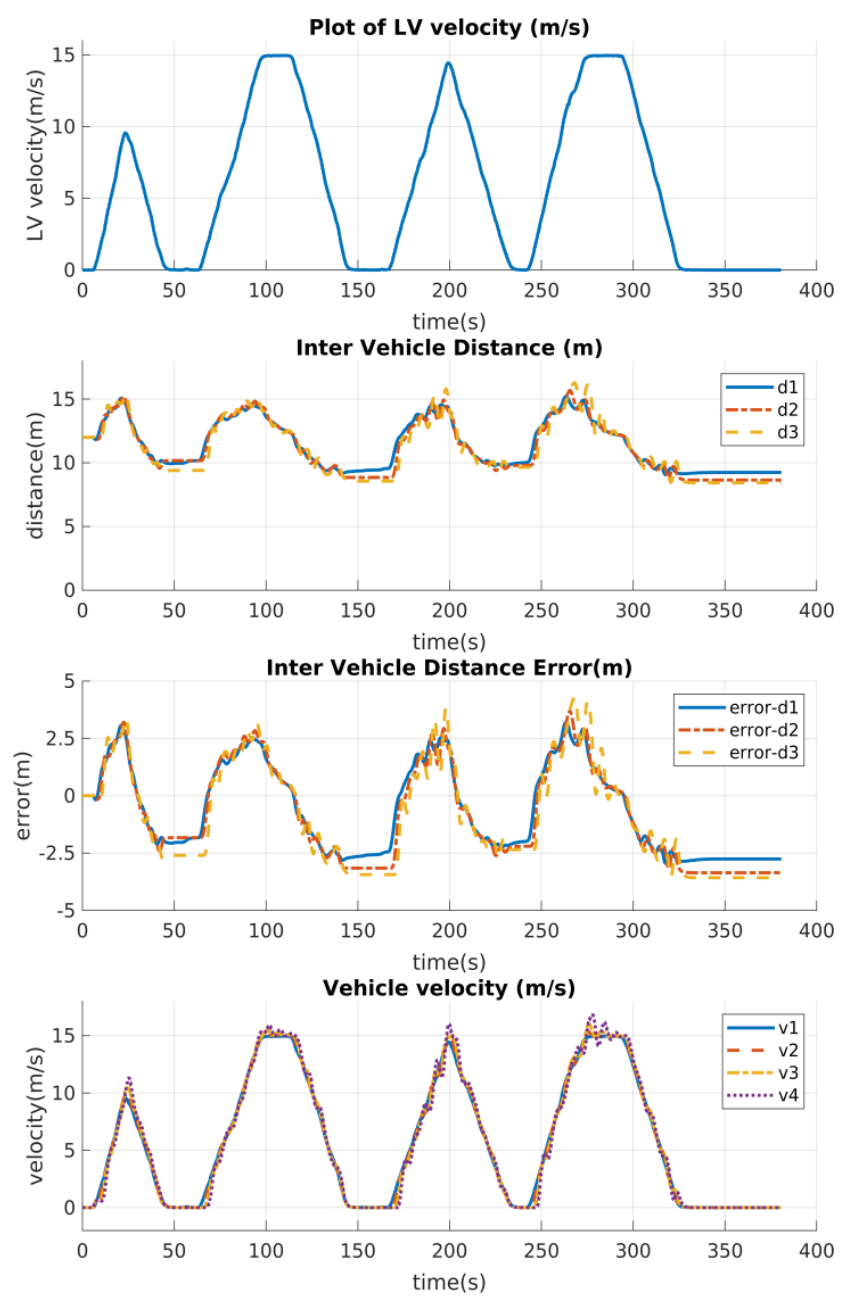

Fig.5. Scenario 1, second case study

in the third subfigure. Finally, the fourth subfigure shows the entire platoon's real-time velocity profiles during the simulation. Just like in, Fig.4. and Fig.5., in the first and second subfigures, blue denotes the $\operatorname{IVD}\left(d_{l}\right)$ and error $\left(E_{l}\right)$ in IVD between the $L V$ and $F_{1}$, respectively. Similarly, red, and green, depict the IVD $\left(d_{2}, d_{3}\right)$ and their corresponding errors $\left(E_{2}, E_{3}\right)$, respectively. In the fourth subfigure, $v 1$, depicted by blue, shows the LV velocity while v2(red), v3(green) and v4(cyan) represent the velocities of the $F$ vehicles (1-3), respectively. In this case study, a minimum error of $-3.8 m$ and a maximum error of $3.1 \mathrm{~m}$ in IVD were obtained with maximum standard deviation and variance of $1.9 \mathrm{~m}$ and $3.7 \mathrm{~m}^{2}$, respectively. During this scenario, local platoon stability is not guaranteed since, for instance, $E_{3} / E_{2}>1$.

In the first subfigure of Scenario 2's second study(Fig.7.), the $L V$ velocity throughout the simulation is presented followed by platoon real-time $\operatorname{IVD}\left(d_{i}\right)$ in the second subfigure. The third subfigure illustrates the $\operatorname{error}\left(E_{i}\right)$ in the IVD in comparison to the desired IVD $(D)$. The fourth subfigure, on the other hand, real-time velocity profiles of the platoon during the simulation. In the first and second subfigures, blue denotes the $\operatorname{IVD}\left(d_{l}\right)$ and error $\left(E_{l}\right)$ in IVD between the $L V$ and $F_{1}$, respectively. Similarly, red and green, denote the IVD $\left(d_{2}\right.$, $\left.d_{3}\right)$ and their corresponding errors $\left(E_{2}, E_{3}\right)$, respectively. In the 

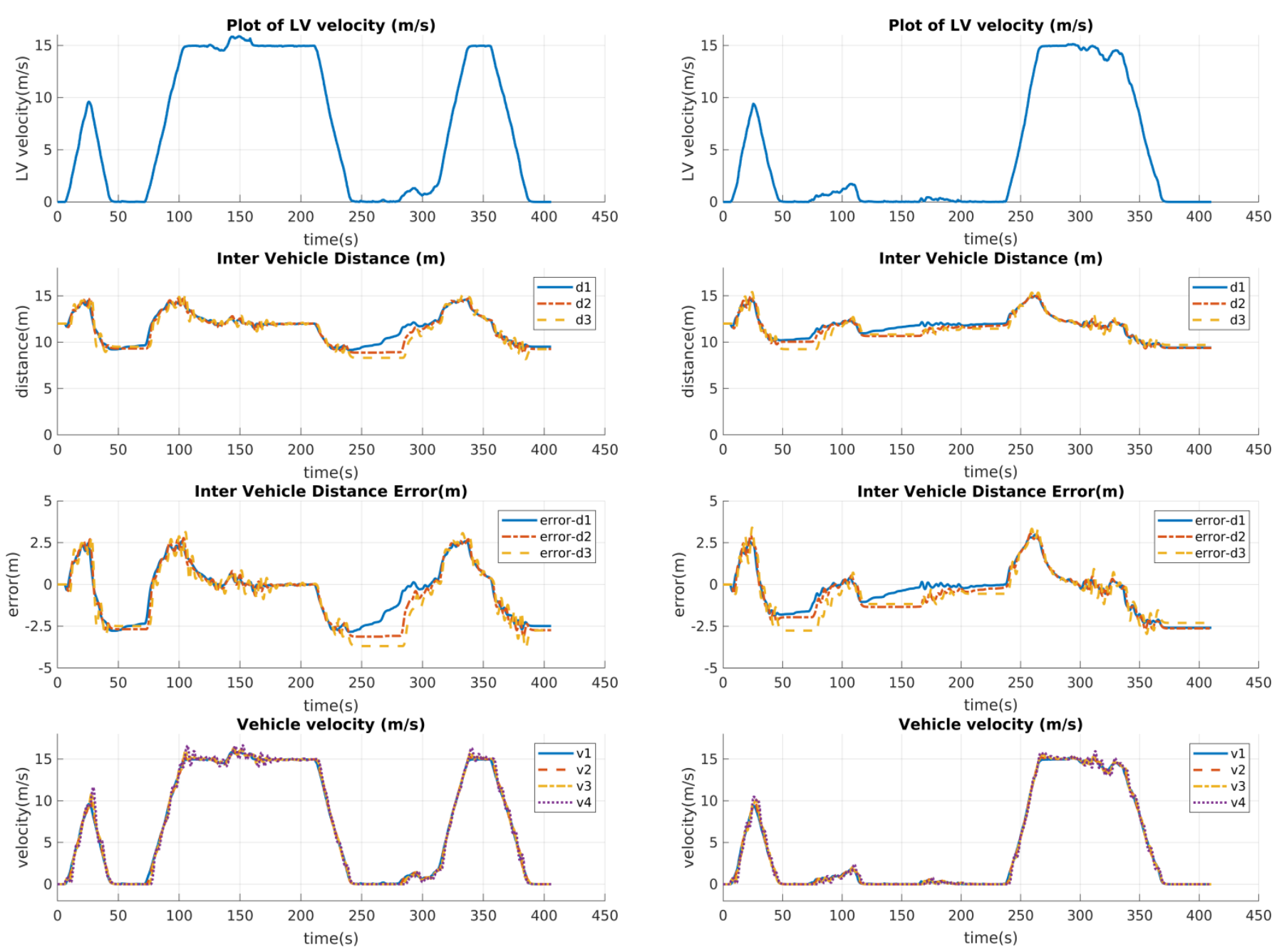

Fig.6. Scenario 2, first case study

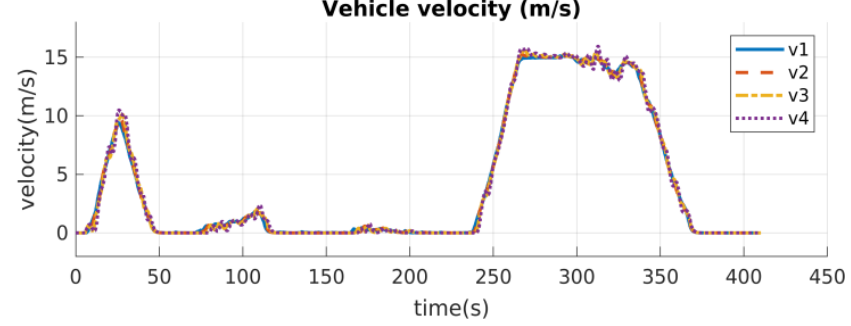

Fig.7. Scenario 2, second case study

fourth subfigure, $v 1$, depicted by blue, shows the LV velocity while v2(red), v3(green) and v4(cyan) represent the velocities of the $F$ vehicles (1-3), respectively. A minimum error of $2.9 \mathrm{~m}$ and a maximum error of $3.4 \mathrm{~m}$ in IVD were obtained with maximum standard deviation and variance of $1.4 \mathrm{~m}$ and $2.0 \mathrm{~m}^{2}$, respectively in this study case. During this scenario, local platoon stability is not guaranteed since, for instance, $E_{3} / E_{2}>$ 1.

\section{Scenario 3}

In this scenario, platoon members were subjected to random V2V communication delays, control delays within the vehicle, and sensor lags without the $H F$ effect.

In the first case study of Scenario 3 (Fig.8.), there is the $L V$ velocity throughout the simulation. Platoon real-time $\operatorname{IVD}\left(d_{i}\right)$ is presented in the second subfigure. The third subfigure presents the error $\left(E_{i}\right)$ in the IVD in comparison to the desired IVD $(D)$. The fourth subfigure presents platoon members' real-time velocity profiles during the simulation. In the first and second subfigures, blue denotes the $\operatorname{IVD}\left(d_{l}\right)$ and error $\left(E_{l}\right)$ in IVD between the $L V$ and $F_{l}$, respectively. Similarly, red and green, denote the IVD $\left(d_{2}, d_{3}\right)$ and their corresponding errors $\left(E_{2}, E_{3}\right)$, respectively. In the fourth subfigure, $v 1$, depicted by

blue, shows the LV velocity while v2(red), v3(green) and v4(cyan) represent the velocities of the $F$ vehicles (1-3), respectively.

During this case study, a minimum error of $-4.4 m$ and a maximum error of $4.9 m$ in IVD were obtained with maximum standard deviation and variance of $1.8 \mathrm{~m}$ and $3.4 \mathrm{~m}^{2}$, respectively. During this scenario, local platoon stability is not guaranteed since, for instance, $E_{2} / E_{1}>1$ almost everywhere. In the second case study of this scenario (Fig.9.), we have the $L V$ velocity throughout the simulation. Platoon real-time $\operatorname{IVD}\left(d_{i}\right)$ is presented in the second subfigure. The third subfigure presents the error $\left(E_{i}\right)$ in the IVD in comparison to the desired IVD $(D)$. The fourth subfigure presents platoon members' real-time velocity profiles during the simulation. In the first and second subfigures, blue denotes the $\operatorname{IVD}\left(d_{l}\right)$ and error $\left(E_{l}\right)$ in IVD between the $L V$ and $F_{l}$, respectively. Similarly, red and green, denote the $\operatorname{IVD}\left(d_{2}, d_{3}\right)$ and their corresponding errors $\left(E_{2}, E_{3}\right)$, respectively. In the fourth subfigure, $v l$, depicted by blue, shows the LV velocity while $\mathrm{v} 2$ (red), v3(green) and v4(cyan) represent the velocities of the $F$ vehicles (1-3), respectively. During the second case study of scenario 3, presented in Fig.9., a minimum error of $-4.1 \mathrm{~m}$ and a maximum error of $4.2 \mathrm{~m}$ in IVD were obtained with maximum standard deviation and variance of $1.9 \mathrm{~m}$ and $3.7 \mathrm{~m}^{2}$, 

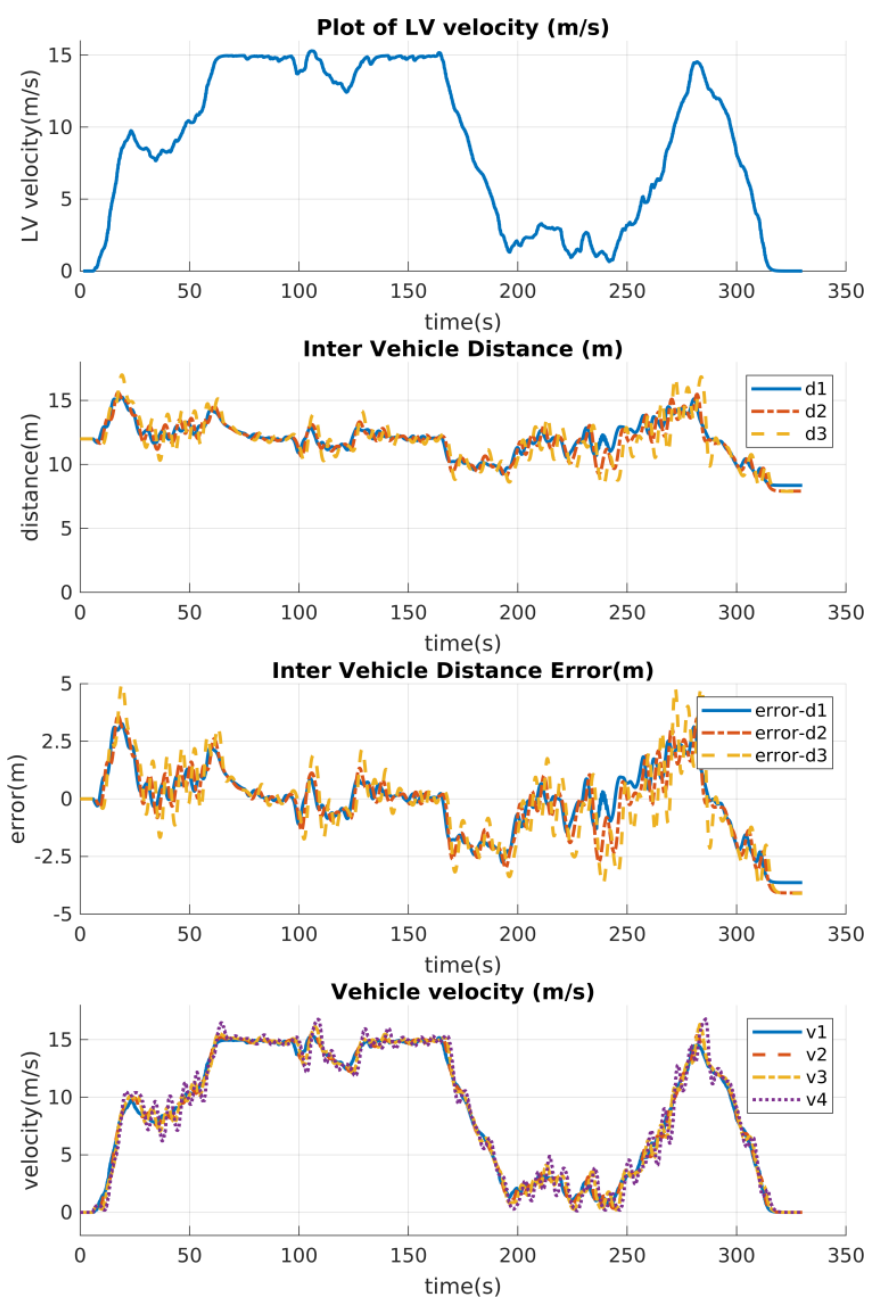

Fig.8. Scenario 3, first case study

respectively. During this scenario, local platoon stability is not guaranteed since, for instance, $E_{3} / E_{2}>1$.

\section{Scenario 4}

In this scenario, the platoon members were subjected to random $\mathrm{V} 2 \mathrm{~V}$ communication and vehicle control delays, sensor lag and random $H F$ effect. In this scenario, platoon stability is not guaranteed, as well, either globally or locally since $H(s)>1$ for all follower vehicles almost everywhere.

In the first subfigure of Fig.10. we present the $L V$ velocity in the simulation. Platoon real-time $\operatorname{IVD}\left(d_{i}\right)$ is presented in the second subfigure. The third subfigure presents the $\operatorname{error}\left(E_{i}\right)$ in the IVD in comparison to the desired IVD $(D)$. The fourth subfigure presents platoon members' real-time velocity profiles during the simulation. In the first and second subfigures, blue denotes the $\operatorname{IVD}\left(d_{l}\right)$ and error $\left(E_{l}\right)$ in IVD between the $L V$ and $F_{1}$, respectively. Similarly, red, and green, denote the $\operatorname{IVD}\left(d_{2}, d_{3}\right)$ and their corresponding errors $\left(E_{2}, E_{3}\right)$, respectively. In the fourth subfigure, $v 1$, depicted by blue, shows the LV velocity while v2(red), v3(green) and v4(cyan) represent the velocities of the $F$ vehicles (1-3), respectively.

During the case study presented in Fig.10. a minimum error of $-3.9 m$ and a maximum error of $3.9 m$ in IVD were obtained
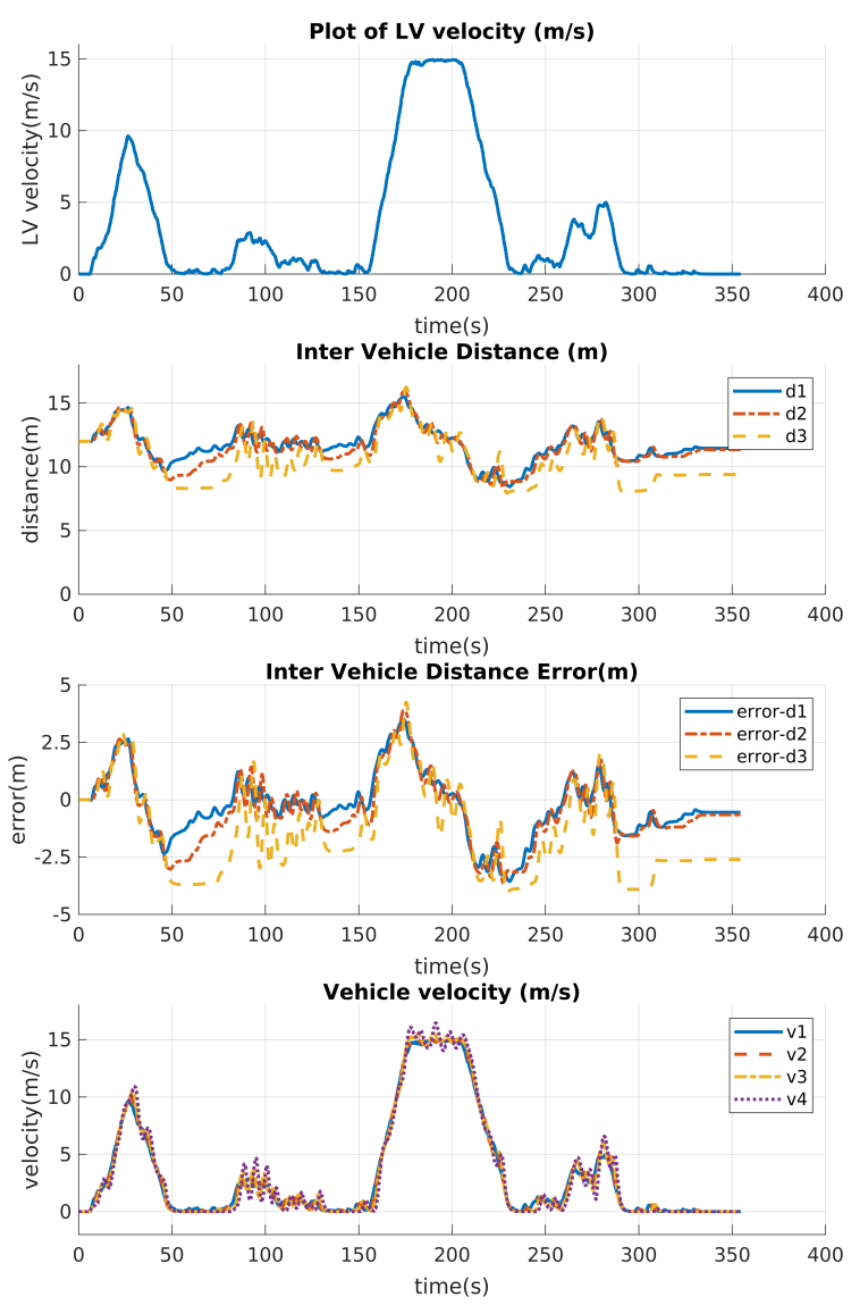

Fig.9. Scenario 3, second case study

with maximum standard deviation and variance of $1.6 \mathrm{~m}$ and $2.6 \mathrm{~m}^{2}$, respectively. During this scenario, local platoon stability is not guaranteed since, for instance, $E_{3} / E_{2}>1$.

Likewise, the first subfigure of Fig.11. demonstrates the $L V$ velocity throughout the simulation. Platoon real-time $\operatorname{IVD}\left(d_{i}\right)$ is presented in the second subfigure. The third subfigure presents the error $\left(E_{i}\right)$ in the IVD in comparison to the desired IVD $(D)$. The fourth subfigure presents platoon members' real-time velocity profiles during the simulation. In the first and second subfigures, blue denotes the $\operatorname{IVD}\left(d_{l}\right)$ and error $\left(E_{l}\right)$ in IVD between the $L V$ and $F_{1}$, respectively. Similarly, red and green, denote the IVD $\left(d_{2}, d_{3}\right)$ and their corresponding errors $\left(E_{2}, E_{3}\right)$, respectively. In the fourth subfigure, $v 1$, depicted by blue, shows the LV velocity while v2(red), v3(green) and v4(cyan) represent the velocities of the $F$ vehicles (1-3), respectively. In the second case study of the fourth scenario (Fig.11.), a minimum error of $-4.5 \mathrm{~m}$ and a maximum error of 4. $1 \mathrm{~m}$ in IVD were obtained with maximum standard deviation and variance of $2.0 \mathrm{~m}$ and $3.9 \mathrm{~m}^{2}$, respectively. During this scenario, local platoon stability is not guaranteed since, for instance, $E_{3} / E_{2}>1$. 

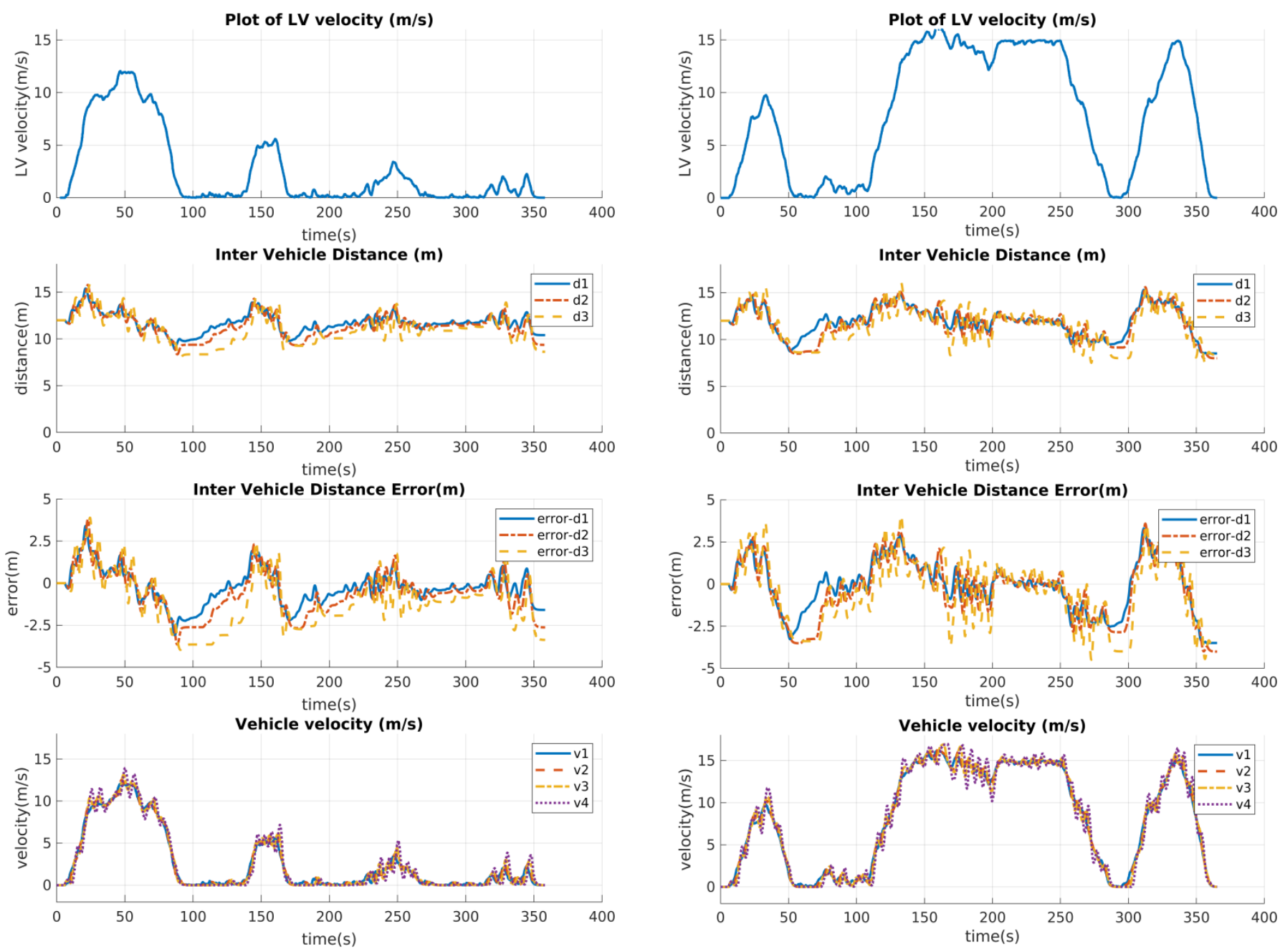

Fig.10. Scenario 4, first case study

\section{DISCUSSION}

In this study, we provide a computationally less demanding longitudinal inter-vehicle distance control algorithm for a platoon of autonomous vehicles that only requires that vehicles be equipped with GPS sensors and have a connection to Wi-Fi. The algorithm computation cost is less than most control algorithms described in the literature. For example, physics-inspired control algorithms such as [22], most of the other PID-based algorithms with different approaches like taking as input to the PID the preceding vehicle's velocity and acceleration e.g., [5] and [23]. Furthermore, this study differs from those that only perform numeric simulations such as [5] by not only using generated GPS data, but also by applying the algorithm to the $3 \mathrm{D}$ models of the platoon to mimic the real world as closely as possible.

However, our approach does have limitations. The first limitation of this algorithm stems from the fact that it is mainly based on GPS sensors. GPS, in reality, is affected by high-frequency faults culminating from multipath errors that occur when signals bounce off surfaces before they can reach the sensor receivers. The position fix, therefore, gets affected as the signals are delayed. Another rarer cause of GPS faults happens when one of the satellites used by the sensor receiver

gets blocked and, as a result, has to be compensated by signals received from a different satellite. The position fix estimated by the GPS sensor is affected by the geometry of the satellites from which the sensor gets signals. So, such changes in configurations of the satellite observed by the sensor receiver affect the position fix finally reported by the GPS receiver.

High-frequency faults and multipath make the accuracy of GPS sensor, and ultimately, the efficiency of our algorithm heavily environment-dependent, making it more accurate and preferable in open space areas than in underground passages, enclosed environments, or places with tall buildings such as skyscrapers. The algorithm can be incorporated into indoor environment or closed environments by replacing the GPS technology with higher precision localization tools and, or sensors such as beacon technology illustrated by [27] and [28].

Autonomous vehicle platooning applications in urban areas involve high precision dependent maneuvers that require about $0.02 m$ accuracy to guarantee safety, among other requirements - such as lane-keeping/changing on busy streets, overtaking operations, to mention but a few. In such applications, a $0.5 \mathrm{~m}$ error is pretty significant. Thus, expanding the applicability of the proposed algorithm to all types of roads and environments requires fusing data from other sensors such as LIDAR, the Inertial Measurement Unit (IMU), and camera. Using sensor fusion to enhance the applicability of the proposed algorithm 
BALKAN JOURNAL OF ELECTRICAL \& COMPUTER ENGINEERING, Vol. 10, No. 1, January 2022

and make it more ideal for even more complex environments is one of our future studies.

\section{CONCLUSION}

In this study, a computationally-cost efficient algorithm for the control of the inter-vehicle distance of autonomous vehicle platoons is presented. The proposed approach takes as input to the PID controller, the updated inter-vehicle distance between a follower vehicle and the preceding vehicle. This distance is calculated from the data measured and provided by the vehicles' onboard GPS sensors. The controller returns the reference velocity with which the follower vehicle should move to achieve the desired inter-vehicle distance. 3D simulations using gazebo and ROS are additionally used to verify and monitor the performance of the system. The proposed approach guarantees the following effect of the platoon ensuring maintenance of platoon formation and no collisions among platoon members. Furthermore, after the transient response to the Leader vehicle's acceleration, the standard deviation of the inter-vehicle distance error was kept under $14.7 \%$ of the desired inter vehicle distance throughout the entire simulation period for all the scenarios. The system seldomly achieved a $\mathbf{0 m}$ error at the steady state when the leader moves with constant speed. However, the proposed method is mainly suitable for open environments since GPS accuracy is susceptible to High-Frequency errors resulting from multipath and collision of GPS signals with surfaces before they reach the receiver. Applicability of the approach can be extended to closed and underground environments if GPS is replaced with high precision localization equipment such as position beacons installed in the target environments. We are currently working on expanding the operability and applicability of the proposed approach on more road types, environments (urban, rural, to mention but a few). Incorporating more sensors and sensor fusion techniques to improve the accuracy of not only the inter-vehicle distance but also the velocity of the platoon members is another direction for our future work.

\section{ACKNOWLEDGMENT}

This work was supported by Istanbul Sabahattin Zaim University's Scientific Research Projects Programme (BAP) ", under Project No: BAP-1001-61.

\section{REFERENCES}

[1] R. Kimura, N. Matsunaga, H. Okajima, and G. Kotaki, "Design of virtual platoon control system using augmented reality to assist welfare vehicle users," International Conference on Control, Automation and Systems, vol. 2017-Octob, no. Iccas, pp. 330-335, 2017..

[2] F. Luo, J. Larson, and T. Munson, "Coordinated platooning with multiple speeds," Transportation Research Part C: Emerging Technologies, vol. 90, pp. 213-225, 2018.

[3] R. Janssen, H. Zwijnenberg, I. Blankers, and J. de Kruijff, "Truck platooning," Driving The Future of Transportation, TNO, 2015.

[4] S. Belcher, E. Merlis, J. McNew, and M. Wright, "Roadmap To Vehicle Connectivity," funded by Crown Castle, Tech. Rep. September, 2018.

[5] V. V. Sivaji and M. Sailaja, "Adaptive cruise control systems for vehicle modeling using stop and go manoeuvres," International Journal of Engineering Research and Applications, vol. 3, no. 4, pp. 2453-2456, 2013.

[Online]. http://citeseerx.ist.psu.edu/viewdoc/download?doi=10.1.1.433.9908\&r=r ep1\&type $=$ pdf

[6] S. E. Shladover, C. Nowakowski, X. Y. Lu, and R. Ferlis, "Cooperative adaptive cruise control: Definitions and operating concepts," Transportation Research Record, vol. 2489, no. November 2014, pp. $145-152,2015$.

[7] G. Naus, R. Vugts, J. Ploeg, R. Van De Molengraft, and M. Steinbuch, "Co-operative adaptive cruise control, design and experiments," Proceedings of the 2010 American Control Conference, ACC 2010, no. 1, pp. 6145-6150, 2010.

[8] D. Swaroop, "String Stability of Interconnected Systems - Automatic Control, IEEE Transactions on," IEEE Transactions on Automatic Control, vol. 41, no. 3, pp. 349 - 357, 1996.

[9] M. Cremer, "On Convoy-Stable Control Laws for Automatically Driven Vehicle Clusters," 1992.

[10] S. Öncü, N. Van de Wouw, W. M. H. Heemels, and H. Nijmeijer, "String stability of interconnected vehicles under communication constraints," in 2012 IEEE 51st IEEE conference on decision and control (cdc). IEEE, 2012, pp. 2459-2464.

[11] T. L. Willke, P. Tientrakool, and N. F. Maxemchuk, "A survey of intervehicle communication protocols and their applications," IEEE Communications Surveys Tutorials, vol. 11, no. 2, pp. 3-20, 2009.

[12] M. Jain and R. Saxena, "Overview of VANET: Requirements and its routing protocols," in 2017 International Conference on Communication and Signal Processing (ICCSP). IEEE, 2017, pp. 1957-1961.

[13] Z. Shen, X. Zhang, D. Yang, I. I. S. Ensor, and S. H. N. Etwork, "Performance Analysis of Extended Sensor Sharing in Vehicular Ad Hoc Networks," 2018 International Symposium on Antennas and Propagation (ISAP), pp. 1-2, 2018.

[14] M. Quigley, "ROS: an open-source Robot Operating System," in ICRA 2009, 2009.

[15] P. Seiler, A. Pant, and K. Hedrick, "Disturbance propagation in vehicle strings," IEEE Transactions on automatic control, vol. 49, no. 10, pp. 1835-1842, 2004.

[16] D. Swaroop, J. K. Hedrick, C. Chien, and P. Ioannou, "A comparision of spacing and headway control laws for automatically controlled vehicles," Vehicle system dynamics, vol. 23, no. 1, pp. 597-625, 1994.

[17] S. Edelkamp, S. Jabbar, and T. Willhalm, "Geometric travel planning," IEEE Conference on Intelligent Transportation Systems, Proceedings, ITSC, vol. 2, no. 1, pp. 964-969, 2003.

[18] S. Sukkarieh, E. M. Nebot, and H. F. Durrant-Whyte, "A high integrity IMU/GPS navigation loop for autonomous land vehicle applications," IEEE Transactions on Robotics and Automation, vol. 15, no. 3, pp. 572578, 1999.

[19] O. Karoui, M. Khalgui, A. Koubâa, E. Guerfala, Z. Li, and E. Tovar, "Dual mode for vehicular platoon safety: Simulation and formal verification," Information Sciences, vol. 402, pp. 216-232, 2017.

[20] E. T. S. Institute, "ETSI TR 103299 V2.1.1 Intelligent Transport Systems (ITS); Cooperative Adaptive Cruise Control (CACC);," Tech. Rep., $2019 . \quad$ [Online]. Available: https://cdn.standards.iteh.ai/samples/45854/7b3e6d0d3c014cb995408e4a 8a0a87d5/ETSI- TR-103-299-V2-1-1-2019-06-.pdf

[21] R. Rajamani and S. E. Shladover, "An experimental comparative study of autonomous and co-operative vehicle-follower control systems," Transportation Research Part C: Emerging Technologies, vol. 9, no. 1, pp.15-31, 2001.

[22] I. Edmunds. (2014) Used 2014 hyundai genesis specs \& features. Used 2014 Hyundai Genesis Specs \& Features. [Online]. Available: https://www.edmunds.com/hyundai/genesis/2014/features-specs/

[23] R. Rajamani, Vehicle dynamics and control. Springer Science \& Business Media, 2011

[24] M. Long, G. Tian, and H. Cheng, "Longitudinal control for truck platooning," in 2020 4th CAA International Conference on Vehicular Control and Intelligence (CVCI), 2020, pp. 418-423.

[25] S.-Y. Yi and K.-T. Chong, "Impedance control for a vehicle platoon system," Mechatronics, vol. 15, no. 5, pp. 627-638, 2005. [Online]. Available:https://www.sciencedirect.com/science/article/pii/S095741580 5000279

[26] Z. Ali Memon, S. Jumani, and J. Larik, "Longitudinal Control of a Platoon of Road Vehicles Equipped with Adaptive Cruise Control System," vol. 31, no. 3, pp. 475-494, 2012. [Online]. Available: http://oaji.net/articles/2016/2712-1454749706.pdf

[27] D. Surian, V. Kim, R. Menon, A. G. Dunn, V. Sintchenko, and E. Coiera, "Tracking a moving user in indoor environments using 
BALKAN JOURNAL OF ELECTRICAL \& COMPUTER ENGINEERING, Vol. 10, No. 1, January 2022

Bluetooth low energy beacons," Journal of Biomedical Informatics, vol. 98, p. 103288, 2019. [Online]. Available: https://www.sciencedirect.com/science/article/pii/S1532046419302072

[28] R. Siegwart and I. R. Nourbakhsh, Introduction to Autonomous Mobile Robots. USA: Bradford Company, 2004.

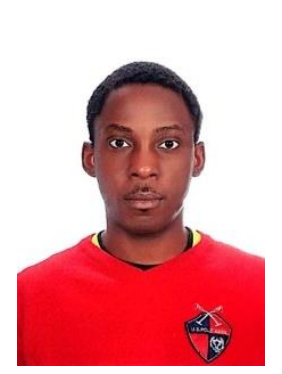

\section{BIOGRAPHIES}

GUNAGWERA ALEX received his M.S. degree in computer science from Istanbul S. Zaim University, Istanbul, Turkey in 2017 where he is currently pursuing the $\mathrm{Ph} . \mathrm{D}$. degree in computer engineering.

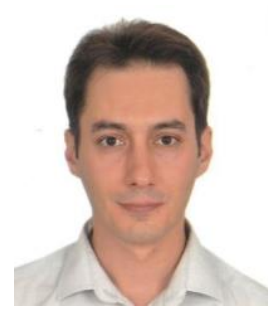

ZENGIN A. TARIK received his electrical and electronics engineering B.S. degree from Ege University, Turkey, in 2007. Later on, received M.E. and Ph.D. degrees from Department of computer science and electrical engineering, Kumamoto University, Japan, in 2010 and 2013, respectively. Currently, he is an Asst. Prof. at Istanbul S. Zaim University. His research interests include Autonomous systems and control theory. 